\title{
The effects of ion adsorption on the potential of zero charge and the differential capacitance of charged aqueous interfaces
}

\author{
Yuki Uematsu ${ }^{1,2}$, Roland R. Netz ${ }^{2}$, and Douwe Jan \\ Bonthuis $^{2}$ \\ 1 Department of Chemistry, Kyushu University, Fukuoka 819-0395, Japan \\ ${ }^{2}$ Fachbereich Physik, Freie Universität Berlin, 14195 Berlin, Germany \\ E-mail: uematsu@chem.kyushu-univ.jp
}

\begin{abstract}
Using different box profile approximations for the non-electrostatic surface adsorption potentials of anions and cations, we calculate the differential capacitance of aqueous electrolyte interfaces from a numerical solution of the Poisson-Boltzmann equation, including steric interactions between the ions and an inhomogeneous dielectric profile. Preferential adsorption of the positive (negative) ion shifts the minimum of the differential capacitance to positive (negative) surface potential values. The trends are similar for the potential of zero charge, however, the potential of zero charge does not correspond to the minimum of the differential capacitance in the case of asymmetric ion adsorption, contrary to the assumption commonly used to determine the potential of zero charge. Our model can be used to obtain more accurate estimates of ion adsorption properties from differential capacitance or electrocapillary measurements. Asymmetric ion adsorption also affects the relative heights of the characteristic maxima in the differential capacitance curves as a function of the surface potential, but even for strong adsorption potentials the effect is small, making it difficult to determine the adsorption properties from the peak heights.
\end{abstract}

Keywords: electrolyte solution, ion adsorption, capacitance

Submitted to: J. Phys.: Condens. Matter 


\section{Introduction}

The adsorption of ions on electrodes and solutes is important for wetting phenomena, colloid stability and electrochemical kinetics [1-3]. Apart from the direct Coulomb interaction between the ions and the surface charges, the ion adsorption potential is strongly influenced by short-ranged Lennard-Jones interactions and dielectric effects, as well as by steric and image charge interactions. Clearly, the consequences of ion adsorption are especially large when anions and cations adsorb asymmetrically - which is typically the case - rendering a net charge to the surface even in the absence of charged surface groups. The potential of zero charge, defined as the surface potential at which the surface charge density vanishes, is therefore a particularly important quantity in the characterization of solid-electrolyte interfaces. In the absence of asymmetric ion adsorption, the potential of zero charge coincides with zero surface potential. Therefore, deviations of this correspondence can be used to study ion adsorption.

There are various ways of measuring the potential of zero charge, often giving disparate results [4]. The most popular experimental method is based on measurements of the differential capacitance of the electric double layer, which characterizes how much charge is stored at an interface between a solid and an electrolyte [5-7]. The capacitance depends on the applied potential in a non-linear fashion, typically showing a minimum between two peaks of differing heights which appear at larger absolute potentials. According to the Gouy-Chapman theory, the minimum of the differential capacitance corresponds to the potential of zero charge. However, the Gouy-Chapman theory does not include surface effects such as specific ion adsorption, and the dependence of the potential of zero charge on the potential of minimum capacitance in the presence of surface effects is yet unclear.

The peaks in the differential capacitance curves have been attributed to steric effects, causing saturation of the double-layer charge, as expressed by the Bikerman equation [8-14]. However, the experimental data show a shift of the potential at the capacitance minimum with increasing salinity, as well as an asymmetric shape with respect to the minimum [5-7], both of which are beyond the assumptions underlying the Bikerman equation. These asymmetric experimental characteristics have been explained by the effect of the different sizes of cations and anions [12] and by the dielectric decrement in the double layer $[13,14]$. Ionic adsorption also affects the asymmetry of the peak heights, which has been used instead of the capacitance minimum to estimate the adsorption potentials $[6,7,15]$. Despite the longstanding interest, a unified model for the analysis of the interfacial capacitance at varying potentials and salt concentrations has been lacking so far.

The ion adsorption potential - comprising all terms except for the direct Coulomb interaction has been calculated using molecular dynamics (MD) simulations [16-18]. For the studied monovalent ions, these potentials of mean force (PMFs) show adsorption or desorption potentials with magnitudes of up to $\sim 2 k_{\mathrm{B}} T$ at several Angstroms from the surface $[18,19]$. In an alternative approach, the structure of water near interfaces has been studied using MD simulations [19-22], revealing that the dielectric constant is highly inhomogeneous near the interface, oscillating and reversing sign repeatedly. This inhomogeneous dielectric profile decreases the differential capacitance because the spatial average of the inverse dielectric profile near the surface is higher than the inverse of the bulk dielectric constant. The differential capacitance at the point of zero charge (PZC) has also been analyzed using this framework for carbons [20,21], but an analysis for finite surface charge density with different surface affinities for cations and anions, as well as a theory for the determination of the $\mathrm{PZC}$, is still lacking.

In this paper, we describe the development of a model of the differential capacitance based on a modified Poisson-Boltzmann equation, where we take ion adsorption into account via step-function approximations of the PMFs. We also take the steric interactions between ions and a dielectric profile into account. With these components, our model contains the essential parameters necessary to study the effects of ion adsorption on the interfacial capacitance and the potential of zero charge. Of course, this simplicity comes at a cost. In particular, interfacial ion densities will not be reproduced accurately, and the model will not be able to precisely reproduce experimental capacitance curves. However, our model shows how the different features observed in experimental data are related to the order of magnitude, the typical length scale and the extent of asymmetry of the ion adsorption and desorption potentials. We find that in the 
presence of asymmetric ion adsorption, the potential at the capacitance minimum differs from the potential of zero charge. Even for mild adsorption potentials of $2 k_{\mathrm{B}} T$, the potentials differ by tens of $\mathrm{mV}$, necessitating a more careful analysis of the experimental data. Asymmetric ion adsorption potentials also affect the asymmetry of the saturation peaks, but the effect is small, making this an inconvenient way to estimate the extent of ion adsorption.

\section{The model}

\subsection{Extended Poisson-Boltzmann equation}

We consider a planar interface $(z=0)$ between an aqueous electrolyte solution $(z>0)$ and a conducting solid or liquid $(z<0)$. All quantities are laterally homogeneous, so the Poisson equation reads [20,21]

$\frac{d}{d z}\left[\varepsilon_{\perp}(z) \varepsilon_{0} \frac{d}{d z} \psi(z)\right]=-\rho(z)$,

where $\varepsilon_{\perp}(z)$ is the local perpendicular dielectric constant, $\varepsilon_{0}$ is the electric permittivity of vacuum, $\psi(z)$ is the local electrostatic potential, and the ionic charge density $\rho(z)$ is given by

$\rho(z)=e\left(c^{+}(z)-c^{-}(z)\right)$,

where $c^{ \pm}(z)$ denote the local concentrations of monovalent cations $(+)$ and anions $(-)$ and $e$ is the elementary charge. Including steric interactions between the ions, the ionic concentration can be written as $[8,11]$

$c^{ \pm}(z)=\frac{c_{\mathrm{salt}}^{\mathrm{b}} \mathrm{e}^{\mp \Psi(z)-U_{ \pm}(z)}}{1+(\nu / 2)\left(\mathrm{e}^{-\Psi(z)-U_{+}(z)}+\mathrm{e}^{\Psi(z)-U_{-}(z)}-2\right)},(3)$

where $c_{\mathrm{salt}}^{\mathrm{b}}$ is the bulk concentration of electrolyte, $\Psi(z)=e \psi(z) / k_{\mathrm{B}} T$ is the dimensionless potential with $k_{\mathrm{B}} T$ being the thermal energy, and $U_{ \pm}(z)$ is the non-electrostatic interaction between the ions and the surface. We set $T=298 \mathrm{~K}$ throughout the paper. The ionic packing parameter $\nu$ is related to the effective ionic diameter $a$ via $\nu=\sqrt{2} c_{\text {salt }}^{\mathrm{b}} a^{3}(<$ 1). This determines the maximum ion concentration $c^{ \pm}=\sqrt{2} / a^{3}$, which is the maximum density of closepacked (face-centered cubic or hexagonal close-packed) spheres of diameter $a$. In the following, models without steric effect are based on (3) with $\nu=0$, and models with steric effects included have a finite $\nu$ based on an estimate for the ionic radius $a$. As the approach of (3) is not applicable to the case of different ion diameters for cations and anions, we use identical diameters in this work. The boundary conditions for (1) are given by

$\left.\varepsilon_{\perp}(z) \varepsilon_{0} \frac{d \psi}{d z}\right|_{z=0}=-\sigma_{0} \quad$ and $\left.\quad \psi(z)\right|_{z \rightarrow+\infty}=0$, with $\sigma_{0}$ being the surface charge density. The additional non-electrostatic ion-surface interaction, which is the primary subject of our present work, is modeled as

$U_{ \pm}(z)= \begin{cases}\alpha_{ \pm} & \text {for } 0<z<z_{\alpha}^{ \pm}, \\ 0 & \text { for } z_{\alpha}^{ \pm}<z,\end{cases}$

where $z_{\alpha}^{ \pm}$is the width of the adsorption layer and $\alpha_{ \pm}$ is the ion-specific surface interaction parameter which determines whether the ion preferentially adsorbs or desorbs from the surface. The non-uniform dielectric constant is also modeled as a box profile [19-24]

$\varepsilon_{\perp}(z)= \begin{cases}\varepsilon_{\text {int }} & \text { for } 0<z<z_{\varepsilon}, \\ \varepsilon & \text { for } z_{\varepsilon}<z,\end{cases}$

where $\varepsilon$ is the dielectric constant of the bulk solution, $\varepsilon_{\text {int }}$ is the dielectric constant in the dielectric interfacial layer and $z_{\varepsilon}$ is the width of the dielectric interfacial layer. We set $\varepsilon=78$ throughout the paper. The extended Poisson-Boltzmann equation in combination with the dielectric profile of (6) has been shown to accurately reproduce the differential capacitance at the potential of zero charge as a function of salt concentration $[20,21,23]$.

\subsection{Parameterization of the box models}

The dielectric profile $\varepsilon_{\perp}(z)$ and the ionic potential of mean force $U_{ \pm}(z)$ have been obtained previously by MD simulations [17, 20,21]. The full dielectric profile $\varepsilon_{\perp}(z)$ is an oscillating function at diamond surfaces [20,21] and between polar soft surfaces [25]. The potential of mean force $U_{ \pm}(z)$ exhibits either a minimum or a monotonic repulsion, depending on ion and surface type [19]. From the full dielectric profile, we determine $z_{\varepsilon}$ and $\varepsilon_{\text {int }}$ as follows [20,21]. Using that in the absence of free charges the displacement field is constant along the $z$ direction (perpendicular to the interface), the differential capacitance $C$ between $z=0$ and $z=z_{\mathrm{b}}$ (in the aqueous bulk phase far from the surface) is given in terms of $\varepsilon_{\perp}(z)$ by

$\frac{1}{C}=\int_{0}^{z_{\mathrm{b}}} \frac{d z}{\varepsilon_{\perp}(z) \varepsilon_{0}}$.

If we use the box profile of (6) for $\varepsilon_{\perp}(z)$, we obtain

$\frac{1}{C}=\frac{z_{\varepsilon}}{\varepsilon_{\mathrm{int}} \varepsilon_{0}}+\frac{z_{\mathrm{b}}-z_{\varepsilon}}{\varepsilon \varepsilon_{0}}$.

Equating (7) and (8) leads to

$z_{\varepsilon}=\int_{0}^{z_{\mathrm{b}}} \frac{\varepsilon_{\perp}(z)^{-1}-\varepsilon^{-1}}{\varepsilon_{\text {int }}^{-1}-\varepsilon^{-1}} d z$.

Similarly, the potential of mean force $U_{ \pm}(z)$ determines the excess number of ions per unit surface area $\Gamma^{ \pm}$, defined as

$$
\begin{aligned}
\Gamma^{ \pm}= & \int_{0}^{z_{\mathrm{GDS}}} c_{\mathrm{salt}}^{\mathrm{b}} \mathrm{e}^{\mp \Psi(z)-U_{ \pm}(z)} d z \\
& +\int_{z_{\mathrm{GDS}}}^{z_{\mathrm{b}}}\left(c_{\mathrm{salt}}^{\mathrm{b}} \mathrm{e}^{\mp \Psi(z)-U_{ \pm}(z)}-c_{\mathrm{salt}}^{\mathrm{b}}\right) d z
\end{aligned}
$$


where $z_{\mathrm{GDS}}$ is the Gibbs dividing surface of water. For a neutral surface and at infinite dilution, the electrostatic contribution $\Psi(z)$ in (10) vanishes, and when using the box profile of (5) for the potential of mean force, we obtain

$\Gamma^{ \pm}=c_{\text {salt }}^{\mathrm{b}}\left(\mathrm{e}^{-\alpha_{ \pm}}-1\right) z_{\alpha}^{ \pm}+c_{\mathrm{salt}}^{\mathrm{b}} z_{\mathrm{GDS}}$.

Again, by equating (10) and (11), we obtain

$z_{\alpha}^{ \pm}=\int_{0}^{z_{\mathrm{b}}} \frac{\mathrm{e}^{-U_{ \pm}(z)}-1}{\mathrm{e}^{-\alpha_{ \pm}}-1} d z$.

Obviously, (9) and (12) are insufficient to determine all free parameters $z_{\varepsilon}, \varepsilon_{\text {int }}, z_{\alpha}^{ \pm}$and $\alpha_{ \pm}$. Moreover, we do not have the full dielectric profiles and the potentials of mean force for all pairs of surfaces and electrolytes. In this paper, therefore, we consider only the case of $z_{\alpha}^{ \pm}=z_{\varepsilon}$ to reduce the number of parameters, and we set $z_{\varepsilon}=0.1 \mathrm{~nm}$, which has been extracted from MD simulations as the width of the layer of low effective dielectric constant for uncharged hydrophilic surfaces [20,21]. Note that these MD simulations have been performed for diamond surfaces with $\varepsilon=1$, whereas capacitance experiments are performed for conducting electrodes. The diamond values still provide a good estimate, however, because similar values of the interfacial capacitance $\varepsilon_{\text {int }} / z_{\varepsilon}$ have been found in simulations of metallic surfaces including image charge effects [26]. Moreover, the effects of $\varepsilon_{\text {int }}$ and $z_{\varepsilon}$ on the shape of the capacitance curves are minor. Finally, image charge effects, which are different at conducting and isolating surfaces, are included in our adsorption parameters $\alpha_{ \pm}$, which we set independently. We assume these interfacial parameters to remain constant when we vary the titrated surface charge density $\sigma_{0}$ and the bulk salt concentration $c_{\text {salt }}^{\mathrm{b}}$. This means that we neglect the dielectric decrement due to the presence of salt $[27,28]$ which has been shown to slightly affect the nonlinear capacitance [14].

In the presence of steric effects and setting $z_{\alpha}^{ \pm}=$ $z_{\varepsilon}$, (1) yields

$\frac{d^{2} \Psi}{d z^{2}}=\left\{\begin{aligned} \frac{\varepsilon \kappa^{2}}{2 \varepsilon_{\text {int }}} \frac{\mathrm{e}^{\Psi-\alpha_{-}}-\mathrm{e}^{-\Psi-\alpha_{+}}}{1+(\nu / 2)\left(\mathrm{e}^{-\Psi-\alpha_{+}}+\mathrm{e}^{\left.\Psi-\alpha_{-}-2\right)}\right.} & \text { for } 0<z<z_{\varepsilon}, \\ \frac{\kappa^{2} \sinh \Psi}{1+\nu(\cosh \Psi-1)} & \text { for } z>z_{\varepsilon},\end{aligned}\right.$

where $\kappa^{-1}=\sqrt{\varepsilon \varepsilon_{0} k_{\mathrm{B}} T / 2 e^{2} c_{\mathrm{salt}}^{\mathrm{b}}}$ is the Debye length.

\section{Differential capacitance}

From the numerical solutions of (13), we calculate the differential capacitance. Apart from the ion distribution in the aqueous phase, the potential variation across the interface between a conducting solid or liquid and an electrolyte is affected by the electron distribution in the conductor. If we assume the contribution of the conductor to the total capacitance to be minor, which is equivalent to assuming a high dielectric constant in the conductor, the differential capacitance is given by

$\frac{1}{C}=\frac{d \psi_{0}}{d \sigma_{0}}$,

with $\psi_{0}$ being the surface potential $\psi(z=0)$. Note, however, that the capacitance of a solid substrate is often finite, and its contribution cannot always be neglected [29-35].

\subsection{Relation between the point of zero charge and the} minimum of the differential capacitance

Before calculating the differential capacitance, we study the relation between the point of zero charge and the minimum of the differential capacitance. In the literature, it is typically assumed that these two points are identical [5-7], but this is not true in general.

From the Gibbs adsorption isotherm, the increment of the surface tension $\gamma$ with the surface potential $\psi_{0}$ and the chemical potentials $\mu_{i}$ of the components obeys

$d \gamma=-\sigma_{0} d \psi_{0}-\sum_{i= \pm} \Gamma_{i} d \mu_{i}$

where $\mu_{ \pm}$are the chemical potentials of cations and anions. We do not consider the variation of the chemical potential of the components, and thus $\gamma$ is a function of the potential difference across the interface denoted by $\psi_{0}$. The condition determining the PZC is $\left.\sigma_{0}\right|_{\mathrm{PZC}}=-\left.\frac{d \gamma}{d \psi_{0}}\right|_{\mathrm{PZC}}=0$,

which corresponds to the zero of the first derivative of $\gamma$. The subscript PZC means the derivative at $\psi_{0}=\psi_{\text {PZC }}$, where $\psi_{\text {PZC }}$ denotes the potential at the PZC. Note that if the cations or anions react with the electrode involving charge transfer, $d \mu_{i}$ is not independent of $d \psi_{0}$ in (15). In this case, the potential of zero total charge is defined by $d \gamma / d \psi_{0}=0$ and distinguished from the potential of zero free charge defined by $\sigma_{0}=0$ [36]. Since we do not consider reactions of ions here, the potentials of zero total charge and zero free charge are identical, and we refer to it as the potential of zero charge. Now, we do a Taylor expansion of $\gamma$ with respect to $\psi_{\mathrm{PZC}}$, yielding

$$
\begin{aligned}
\gamma\left(\psi_{0}\right)= & \gamma\left(\psi_{\mathrm{PZC}}\right)+\left.\frac{1}{2} \frac{d^{2} \gamma}{d \psi_{0}^{2}}\right|_{\mathrm{PZC}}\left(\psi_{0}-\psi_{\mathrm{PZC}}\right)^{2} \\
& +\left.\frac{1}{6} \frac{d^{3} \gamma}{d \psi_{0}^{3}}\right|_{\mathrm{PZC}}\left(\psi_{0}-\psi_{\mathrm{PZC}}\right)^{3} \\
& +\left.\frac{1}{24} \frac{d^{4} \gamma}{d \psi_{0}^{4}}\right|_{\mathrm{PZC}}\left(\psi_{0}-\psi_{\mathrm{PZC}}\right)^{4}+\cdots
\end{aligned}
$$


Note that the term linear in $\psi_{0}-\psi_{\mathrm{PZC}}$ in (17) does not appear by virtue of (16). The differential capacitance is by definition

$C\left(\psi_{0}\right)=\frac{d \sigma_{0}}{d \psi_{0}}=-\frac{d^{2} \gamma}{d \psi_{0}^{2}}$,

and using (17), we have

$$
\begin{aligned}
C\left(\psi_{0}\right)= & -\left.\frac{d^{2} \gamma}{d \psi_{0}^{2}}\right|_{\mathrm{PZC}}-\left.\frac{d^{3} \gamma}{d \psi_{0}^{3}}\right|_{\mathrm{PZC}}\left(\psi_{0}-\psi_{\mathrm{PZC}}\right) \\
& -\left.\frac{1}{2} \frac{d^{4} \gamma}{d \psi_{0}^{4}}\right|_{\mathrm{PZC}}\left(\psi_{0}-\psi_{\mathrm{PZC}}\right)^{2}+\cdots
\end{aligned}
$$

Equation (19) is the Taylor expansion of $C\left(\psi_{0}\right)$ with respect to $\psi_{\mathrm{PZC}}$. The potential of the minimum or maximum capacitance close to the PZC is approximately equal to

$\psi_{\mathrm{m}} \approx \psi_{\mathrm{PZC}}-\left.\frac{d^{3} \gamma / d \psi_{0}^{3}}{d^{4} \gamma / d \psi_{0}{ }^{4}}\right|_{\mathrm{PZC}}$,

which shows that the minimum of the differential capacitance generally does not correspond to the potential at the PZC.

\subsection{Differential capacitance at finite surface charge}

First, we calculate the differential capacitance for symmetric ion adsorption potentials $\left(\alpha_{+}=\alpha_{-}\right)$. We use $z^{*}=0.1 \mathrm{~nm}$, and $\varepsilon_{\text {int }}=1$ which are extracted from molecular dynamics simulations of hydrophilic surfaces [20,21]. The results for $\alpha_{ \pm}=0,2$, and -2 are shown in figure 1 . Without adsorption potentials $\left(\alpha_{+}=\alpha_{-}=0\right)$, the capacitance curves show two maxima for low salt concentration, whereas for large salt concentration, the two maxima move close to each other and finally form a single maximum. As shown in figure $1 \mathrm{~d}$, the height of the maxima for $c_{\mathrm{salt}}^{\mathrm{b}} \leq$ $100 \mathrm{mM}$ is almost independent of the salt concentration (black triangles). Because of the symmetric adsorption $\left(\alpha_{+}=\alpha_{-}\right)$, the potentials at the minimum or the single maximum of the capacitance curves (defined by $\left.\psi_{\mathrm{m}}\right)$ are identical to the potentials of zero charge $\psi_{\mathrm{PZC}}$ for all cases $\left(\alpha_{ \pm}=0,2,-2\right)$. When both cations and anions are repelled $\left(\alpha_{+}=\alpha_{-}=2\right)$, as shown in figure $1 b$, the shape of the capacitance curves becomes broader compared to the case $\left(\alpha_{+}=\alpha_{-}=0\right)$. By contrast, when both cations and anions are adsorbed $\left(\alpha_{+}=\alpha_{-}=-2\right)$, as shown in figure 1c, the parabolic shape of the capacitance curves becomes narrower compared to the case $\left(\alpha_{+}=\alpha_{-}=0\right)$. As shown in figure $1 \mathrm{~d}$, we find that the repulsion $\left(\alpha_{+}=\alpha_{-}=2\right)$ decreases and the adsorption $\left(\alpha_{+}=\alpha_{-}=2\right)$ increases the height of the capacitance maxima on both sides of the minimum, but only by a negligibly small amount for small concentration $\left(c_{\text {salt }}^{\mathrm{b}} \leq 100 \mathrm{mM}\right)$.

Second, we study the case of asymmetric adsorption, $\alpha_{+} \neq \alpha_{-}$. In figure $2 \mathrm{a}$, we show
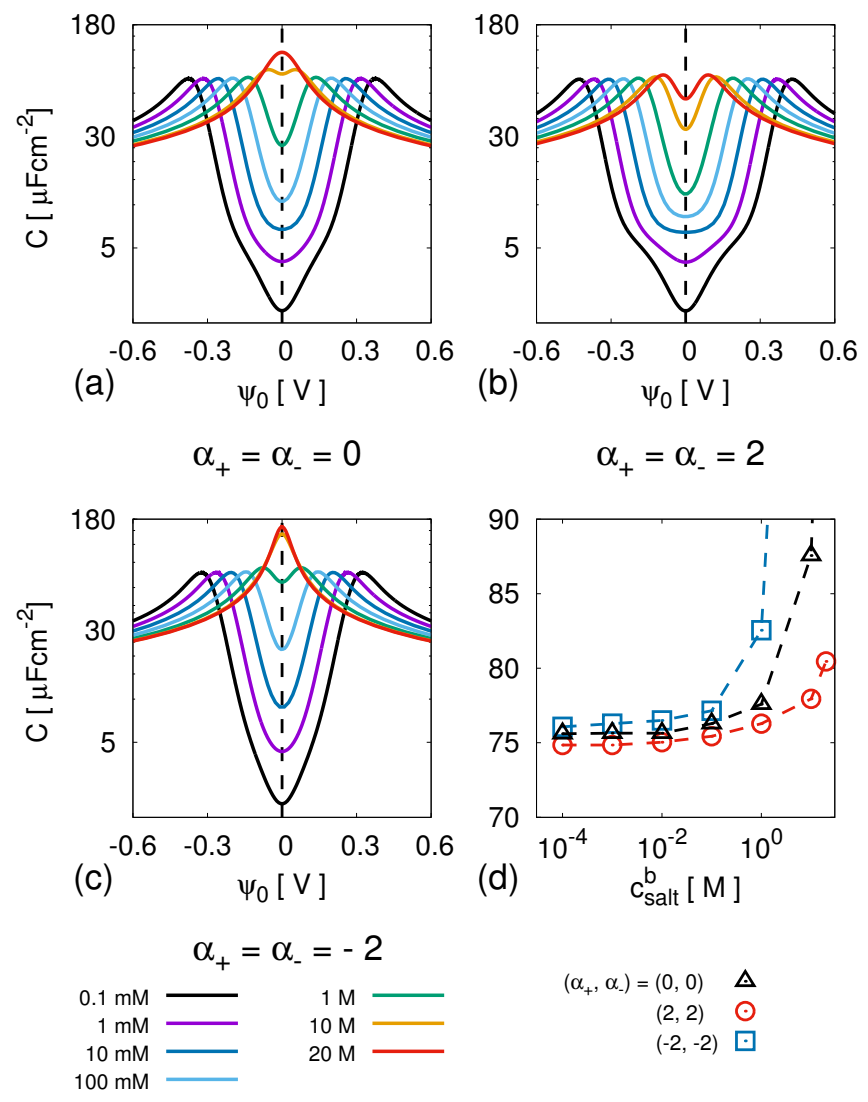

Figure 1. (a,b,c) Differential capacitance as a function of the surface potential. We use $z_{\varepsilon}=0.1 \mathrm{~nm}, \varepsilon_{\text {int }}=1$, and $a=0.3 \mathrm{~nm}$. The affinity parameters are (a) $\alpha_{+}=\alpha_{-}=0$, (b) $\alpha_{+}=\alpha_{-}=2$, and (c) $\alpha_{+}=\alpha_{-}=-2$. (d) The peak values of the differential capacitance as a function of salt concentration. The broken lines are guides to eyes.

the differential capacitance for $\alpha_{+}=2, \alpha_{-}=0$ (cation more repulsive than anion), calculated using a numerical solution to (13). The position of the capacitance minimum $\psi_{\mathrm{m}}$ moves to more negative values, as expected. As shown in figure $2 \mathrm{~b}$, the heights of the maxima for negative $\psi_{0}$ (open squares) slightly decrease compared to those for $\psi_{0}>0$ (filled squares), although for $c_{\text {salt }}^{\mathrm{b}}>10 \mathrm{M}$ the difference is indistinguishable. In figure $2 \mathrm{c}$, we plot $\psi_{\mathrm{m}}$ and $\psi_{\mathrm{PZC}}$, both of which are decreasing as a function of salt concentration. However, the $\psi_{\mathrm{m}}$ and $\psi_{\mathrm{PZC}}$ differ by several tens of $\mathrm{mV}$ in the range of $1 \mathrm{mM}<c_{\text {salt }}^{\mathrm{b}}<1 \mathrm{M}$, which shows that the difference derived in $(20)$ is nonnegligible in this case. The red broken lines in figure $2 \mathrm{a}, \mathrm{c}$ denote the potential

$\psi_{0}=-\frac{k_{\mathrm{B}} T}{e} \frac{\alpha_{+}-\alpha_{-}}{2}$,

which is a special surface potential where the charge density (2) near the surface $(z \rightarrow+0)$ vanishes. Equation (21) has the same sign as $\psi_{\mathrm{m}}$ and $\psi_{\mathrm{PZC}}$, and both $\psi_{\mathrm{m}}$ and $\psi_{\mathrm{PZC}}$ approach (21) at large salt concentration. In figure $2 \mathrm{~d}$, we plot the case $\alpha_{+}=-2$ 

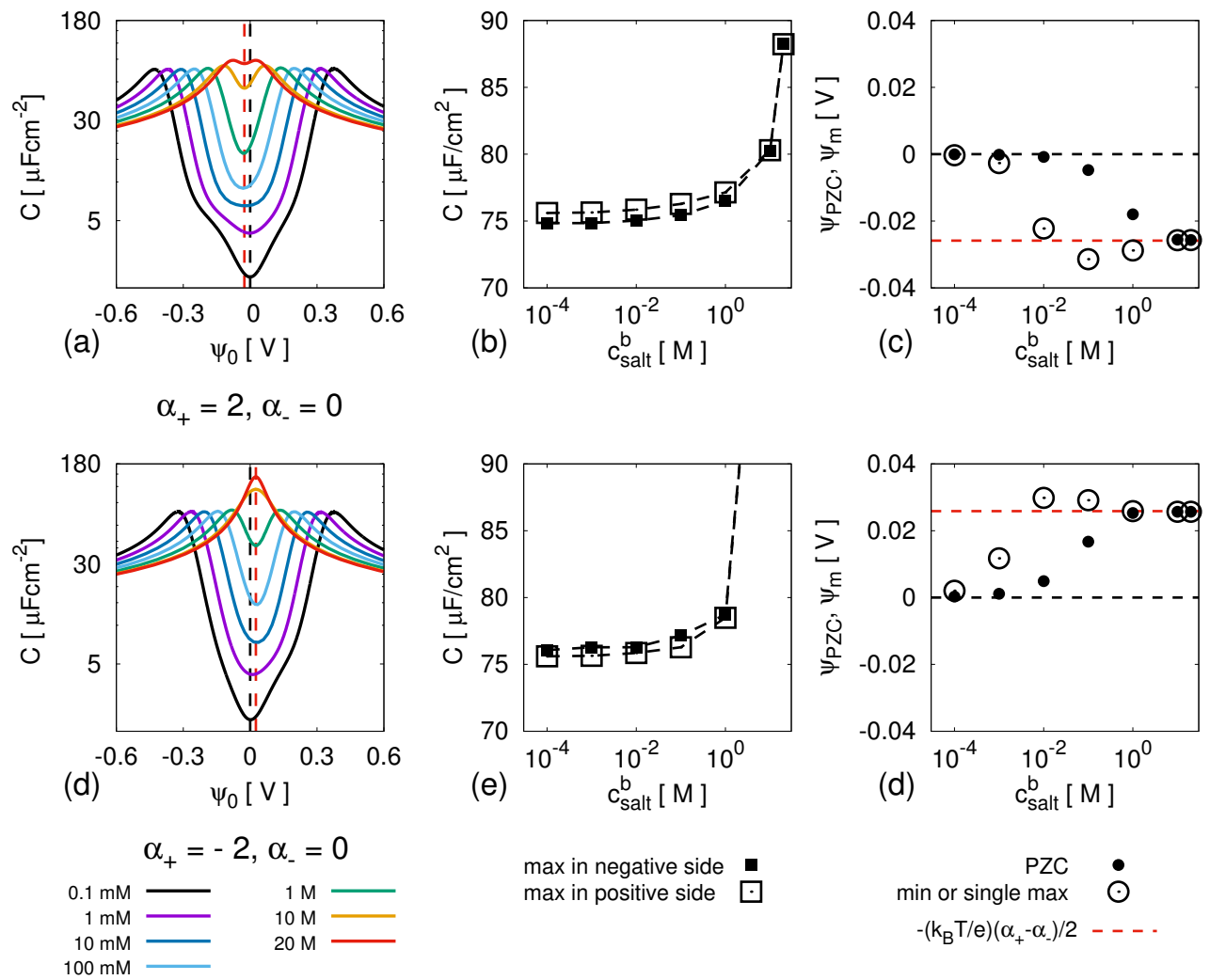

Figure 2. (a) Differential capacitance as a function of the surface potential $\psi_{0}$. We use $z_{\varepsilon}=0.1 \mathrm{~nm}, \varepsilon_{\text {int }}=1$, and $a=0.3 \mathrm{~nm}$. The affinity parameters are $\alpha_{+}=2$ and $\alpha_{-}=0$. (b) The values of the peaks in the capacitance curves as a function of salt concentration for $\alpha_{+}=2$ and $\alpha_{-}=0$. (c) $\psi_{\mathrm{m}}$ and $\psi_{\mathrm{PZC}}$ are plotted as a function of salt concentration for $\alpha_{+}=2$ and $\alpha_{-}=0$. (d) Differential capacitance as a function of the surface potential for $\alpha_{+}=-2$ and $\alpha_{-}=0$. (e) The values of the peaks in the capacitance curves as a function of salt concentration. The affinity parameters are $\alpha_{+}=-2$ and $\alpha_{-}=0$. (f) $\psi_{\mathrm{m}}$ and $\psi_{\mathrm{PzC}}$ are plotted as a function of salt concentration for $\alpha_{+}=-2$ and $\alpha_{-}=0$.

and $\alpha_{-}=0$ (cation more adsorbing than anion). The position of the minimum shifts to more positive values with increasing salt concentration, reflecting the cation adsorption. In this case, the heights of the maxima for $\psi_{0}<0$ are decreased, compared to those for $\psi_{0}>0$ (see figure 2e). Figure $2 \mathrm{f}$ shows that $\psi_{\mathrm{m}}$ and $\psi_{\mathrm{PZC}}$ are both increasing functions of $c_{\text {salt }}^{\mathrm{b}}$, but again, their values are different and both approach (21) at large salt concentration. The absolute deviations between $\psi_{\mathrm{m}}$ and $\psi_{\mathrm{PZC}}$ show a similar trend for cation desorption (figure 2a-c) and cation adsorption (figure 2d-f), but their magnitudes depend intricately on the salt concentration, as well as on the adsorption asymmetry $\left(\alpha_{+}-\alpha_{-}\right)$and even on the individual values of $\alpha_{+}$and $\alpha_{-}$. Therefore, over a very large range of salt concentrations, an accurate determination of the potential of zero charge from the differential capacitance requires explicit knowledge of the ion adsorption potentials in the interfacial model.

Because the height of the maxima are not affected so much by weak affinities $\alpha_{i}= \pm 2$, we also examine the effects of strong affinities $\alpha_{i}= \pm 10$, which is significantly stronger than those extracted from potentials mean force of simple monovalent ions in MD simulations [16-19]. Figure 3a shows the differential capacitance for $\alpha_{+}=10$ and $\alpha_{-}=0$. Like in figure $2 \mathrm{a}, \psi_{\mathrm{m}}$ moves to the negative side. Furthermore, the maximum on the positive side becomes higher than on the negative side (see figure $3 \mathrm{~b}$ ). However, the difference of $\sim 5 \mu \mathrm{F} / \mathrm{cm}^{2}$, which is induced here only by the asymmetry in the adsorption potentials of cations and anions, is negligible compared to the variation of the capacitance found in experiments for different salt types [5-7]. This implies that the large asymmetry of the capacitance curves in experiments is caused by other effects, for example by asymmetric steric interactions between the ions or by changes of the dielectric constant due to added ions [13,14], which are not studied in this paper. In figure $3 \mathrm{~d}$, the differential capacitance for $\alpha_{+}=-10$ and $\alpha_{-}=0$ is plotted, and $\psi_{\mathrm{m}}$ moves to the positive side. The increase of the heights of the maxima for $\psi_{0}<0$ are small, but the two maxima move closer to each other and the height increases for large salt concentration. In figure $3 \mathrm{c}, \mathrm{f}$, we plot $\psi_{\mathrm{m}}$ and $\psi_{\mathrm{PZC}}$, showing qualitatively similar features to those in figure $2 c, f$. 

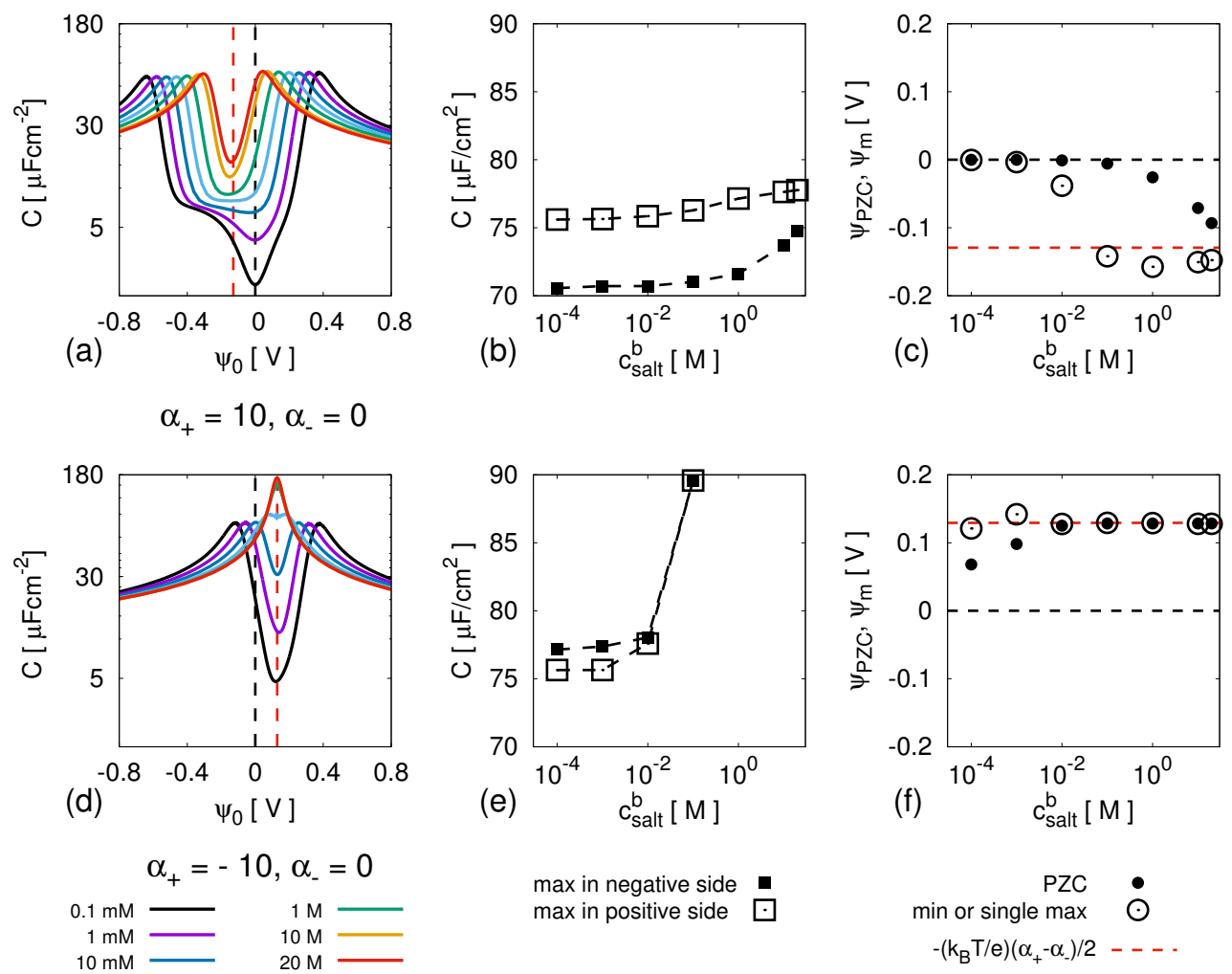

Figure 3. (a) Differential capacitance as a function of the surface potential. We use $z_{\varepsilon}=0.1 \mathrm{~nm}, \varepsilon_{\text {int }}=1$, and $a=0.3 \mathrm{~nm}$. The affinity parameters are $\alpha_{+}=10$ and $\alpha_{-}=0$. (b) The values of the peaks in the capacitance curves as a function of salt concentration for $\alpha_{+}=10$ and $\alpha_{-}=0$. (c) $\psi_{\mathrm{m}}$ and $\psi_{\mathrm{PZC}}$ are plotted as a function of salt concentration for $\alpha_{+}=10$ and $\alpha_{-}=0$. (d) Differential capacitance as a function of the surface potential for $\alpha_{+}=-10$ and $\alpha_{-}=0$. (e) The values of the peaks in the capacitance curves as a function of salt concentration. The affinity parameters are $\alpha_{+}=-10$ and $\alpha_{-}=0$. (f) $\psi_{\mathrm{m}}$ and $\psi_{\mathrm{PZC}}$ are plotted as a function of salt concentration for $\alpha_{+}=-10$ and $\alpha_{-}=0$.

\section{Conclusions}

When anions and cations have different surface affinities, the potential of zero charge and the potential at which the capacitance has a minimum, $\psi_{\mathrm{m}}$, have the same sign. In the limit of low salt concentration both potentials are zero whereas in the limit of large salt concentration both approach $-\left(k_{\mathrm{B}} T / e\right)\left(\alpha_{+}-\alpha_{-}\right) / 2$. However, in the intermediate concentration range $1 \mathrm{mM}$ to $1 \mathrm{M}$, they have different values. Therefore, the common method to determine of the potential of zero charge from the minimum in the differential capacitance is inaccurate in the presence of asymmetric ion adsorption. The deviation between $\psi_{\mathrm{m}}$ and $\psi_{\mathrm{PZC}}$ depends intricately on the salt concentration and the ion adsorption potentials. Numerical solutions of our simple model can be used to extract a more accurate estimate of the ion adsorption potentials from differential capacitance curves, ideally combined with electrocapillary curves $\gamma\left(\psi_{0}\right)$.

Coion-repulsion decreases the height of the capacitance maxima, but for weak repulsion $\left(\alpha_{i}=2\right)$ the change is negligible, and for strong repulsion $\left(\alpha_{i}=\right.$
10) it goes down by approximately $5 \mu \mathrm{F} / \mathrm{cm}^{2}$. Coionadsorption increases the height of the maxima a little bit for both weak and strong adsorption $\left(\alpha_{i}=-2\right.$ and $-10)$, but for large salt concentration $\left(c_{\text {salt }}^{\mathrm{b}} \geq 1 \mathrm{M}\right)$ the two maxima move close to each other, their height increases, and finally the capacitance curve shows a single maximum. Overall, the change of the peak heights for small concentration $\left(c_{\text {salt }}^{\mathrm{b}} \leq 100 \mathrm{mM}\right)$ is not very sensitive to the underlying ion adsorption potentials, and negligible in comparison to the effects typically found in experiments. This suggests that the large difference of the two maximum capacitances found in experiments is most likely caused by other effects such as asymmetric ion steric interactions or variations of the dielectric constant due to added ions. Direct comparison of our curves with experimental data is not straightforward because we neglect the contributions of the substrate capacitance, different ionic sizes of cations and anions, and the dielectric decrement with salt concentration. In this paper we concentrate on the effect of ion adsorption and repulsion on the potential of zero charge and the differential capacitance only. 


\section{Acknowledgments}

R.R.N. and D.J.B acknowledge support from the DFG project NE 810/10-1. Y.U. acknowledges support from Grant-in-Aid for JSPS Fellows 16J00042.

\section{References}

[1] Dukhin S S and Derjaguin B V 1974 Surafce and Colloid Science vol 7 (New York: Wiley)

[2] Russel W B, Saville D A and Schowalter W R 1989 Colloidal Dispersions (Cambridge: Cambridge University Press)

[3] Lyklema J 1995 Fundamentals of Interface and Colloid Science vol 3 (London: Academic Press)

[4] Bockris J O, Argade S D and Gilead E 1969 Electrochim. Acta 14 1259-1283

[5] Grahame D C 1947 Chem. Rev. 41 441-501

[6] Valette G 1981 J. Electroanal. Chem. 122 285-297

[7] Valette G $1982 \mathrm{~J}$. Electroanal. Chem. 138 37-54

[8] Bikerman J J 1942 Philos. Mag. 733 384-397

[9] Eigen M and Wicke E 1954 J. Phys. Chem. 58 702-714

[10] Kralj-Iglič V and Iglič A 1994 Electrotech. Rev. Ljubljana, Slovenija 61 127-133

[11] Borukhov L, Andelman D and Orland H 1997 Phys. Rev. Lett. 79435

[12] Kilic M S, Bazant M Z and Ajdari A 2007 Phys. Rev. E 75 021502

[13] Hatlo M M, van Roji R and Lue L 2012 EPL 9728010

[14] Nakayama Y and Andelman D 2015 J. Chem. Phys. 142 044706

[15] Devanathan M A V 1954 Trans. Faraday. Soc. 50 373-385

[16] Jungwirth P and Tobias D J 2006 Chem. Rev. 106 12591281

[17] Horinek D and Netz R R 2007 Phys. Rev. Lett. 99226104

[18] Mamatkulov S I, Allolio C, Netz R R and Bonthuis D J 2017 Angew. Chem. Int. Ed.

[19] Bonthuis D J and Netz R R 2013 J. Phys. Chem. B 117 11397-11413

[20] Bonthuis D J, Gekle S and Netz R R 2011 Phys. Rev. Lett. 107166102

[21] Bonthuis D J, Gekle S and Netz R R 2012 Langmuir 28 7679

[22] Bonthuis D J and Netz R R 2012 Langmuir 28 16049-16059

[23] Bonthuis D J, Uematsu Y and Netz R R 2016 Phil. Trans. R. Soc. A $\mathbf{3 7 4} 20150033$

[24] Uematsu Y, Netz R R and Bonthuis D J 2017 Chem. Phys. Lett. $67011-15$

[25] Schlaich A, Knapp E W and Netz R R 2016 Phys. Rev. Lett. 117048001

[26] Willard A P, Reed S K, Madden P A and Chandler D 2009 Faraday Discuss. $141423-441$

[27] Rinne K, Gekle S and Netz R R 2014 J. Phys. Chem. A $11811667-11677$

[28] Rinne K, Gekle S and Netz R R 2014 J. Chem. Phys. 141 214502

[29] Schmickler W and Henderson D 1984 J. Chem. Phys. 80 $3381-3386$

[30] Gerischer H 1985 J. Phys. Chem. 89 4249-4251

[31] Schmickler W and Henderson D 1986 J. Chem. Phys. 85 1650-1657

[32] Gerischer H, McIntyre R, Scherosn D and Storck W 1987 J. Phys. Chem. 91 1930-1935

[33] Schmickler W 1996 Chem. Rev. 96 3177-3200

[34] Luque N B and Schmickler W 2012 Electrochim. Acta 71 $82-85$

[35] Federov M V and Kornyshev A A 2014 Chem. Rev. 114 2978-3036
[36] Trasatti S and Lust E 1999 Modern aspects of electrochemistry vol 33 (New York: Kluwer) 